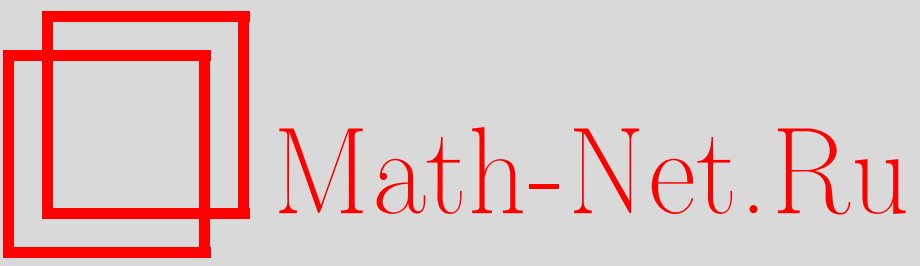

Yu. Davydov, V. Paulauskas, A simple approach in limit theorems for linear random processes and fields with continuous time, Теория вероятн. и ее примен., 2012, том 57, выпуск 4, 724-743

DOI: https://doi.org/10.4213/tvp4476

Использование Общероссийского математического портала Math-Net.Ru подразумевает, что вы прочитали и согласны с пользовательским соглашением

http://www . mathnet.ru/rus/agreement

Параметры загрузки:

IP: 18.207 .199 .55

26 апреля 2023 г., 15:14:32 
ТЕОРИЯ ВЕРОЯТНОСТЕЙ

Том 57

И ЕЕ ПРИМЕНЕНИЯ

Выпуск 4

2012

(c) 2012 г. DAVYDOV YU.* , PAULAUSKAS V.**

\title{
A SIMPLE APPROACH IN LIMIT THEOREMS FOR LINEAR RANDOM PROCESSES AND FIELDS WITH CONTINUOUS TIME ${ }^{1)}$
}

\begin{abstract}
Рассматриваются линейные случайные процессы и поля с непрерывным временем или пространственным аргументом и показывается, как аналог разложения Бевериджа-Нельсона может быть применен при доказательстве предельных теорем для интегралов от таких процессов и полей.

Ключевые слова и фразы: предельные теоремы, линейные случайные процессы и поля, разложение Бевериджа-Нельсона.
\end{abstract}

1. Introduction. In May 2008 in Luminy at the conference «High dimensional probability» the second author gave a talk on some decomposition for linear random fields on $\mathbf{Z}^{d}$ and application of this decomposition to limit theorems for such fields. The talk was based on [8], the results of which can be considered as rather straightforward generalization of the results for time series from the paper by Phillips and Solo [9]. This decomposition for linear processes was invented (more precisely, reinvented, see remarks below) in purely applied econometric paper [3], and in econometric literature is called Beveridge-Nelson decomposition (BND). Jan Rosinski asked if such decomposition exists for linear random processes and fields with arguments in $\mathbf{R}$ and $\mathbf{R}^{d}$, respectively. In the paper we present some analogue of this decomposition in continuous time.

First we shall remind original BND for linear processes with discrete time. Let

$$
X_{t}=\sum_{k=0}^{\infty} c_{k} \varepsilon_{t-k}, \quad t \in \mathbf{Z}
$$

be a linear process, where $\left\{\varepsilon_{i}, i \in \mathbf{Z}\right\}$ is a stationary sequence and $c_{i}$ and $\varepsilon_{i}$ are such that $X_{t}$ is correctly defined (series converges a.s.) and, therefore, is a stationary process. In time series theory such processes are called

\footnotetext{
${ }^{*}$ Université des Sciences et Technologies de Lille, Laboratoire Paul Painlevé, Lille, France.

${ }^{* *}$ Department of Mathematics and Informatics, Vilnius University, Vilnius, Lithuania; e-mail: vygantas.paulauskas@mif.vu.lt

1) The research was supported by the bilateral France-Lithuania scientific project Gilibert.
} 
causal, since the value of the process $X_{t}$ is determined by the values of the innovations $\varepsilon_{t-k}, k \geqslant 0$, that is, in the past. More general linear processes with summation in (1) over all $\mathbf{Z}$ will be considered after Proposition 2. As sufficient conditions for the correctness of definition of $X_{t}$ one can provide the following ones: $\sum_{k=0}^{\infty}\left|c_{k}\right|<\infty$ and $\mathbf{E}\left|\varepsilon_{0}\right|<\infty$. Dealing with BND we shall always assume that the condition $\sum_{k=0}^{\infty}\left|c_{k}\right|<\infty$ is satisfied, and we denote $C_{1}=\sum_{k=0}^{\infty} c_{k}$. Main cases in the theory of linear processes are the cases where $\left\{\varepsilon_{i}, i \in \mathbf{Z}\right\}$ are independent and identically distributed (i.i.d.) random variables or martingale differences. There are several approaches to investigate sums of (dependent) random variables $\sum_{t=1}^{n} X_{t}$, one of them is based on the above mentioned BND of linear processes. This decomposition being purely algebraic, it is easy to formulate the following proposition.

Proposition 1 (see [3], [9]). Assume that all quantities are defined correctly, then the following identity holds:

$$
\sum_{k=0}^{\infty} c_{k} \varepsilon_{t-k}=\varepsilon_{t} C_{1}+\tilde{\varepsilon}_{t-1}-\tilde{\varepsilon}_{t}
$$

where $\tilde{\varepsilon}_{t}=\sum_{k=0}^{\infty} \tilde{c}_{k} \varepsilon_{t-k}, \tilde{c}_{k}=\sum_{j=k+1}^{\infty} c_{j}$. If $p \geqslant 1$, then

$$
\sum_{j=1}^{\infty} j^{p}\left|c_{j}\right|^{p}<\infty \Rightarrow \sum_{k=0}^{\infty}\left|\tilde{c}_{k}\right|^{p}<\infty \quad \text { and } \quad\left|\mathrm{C}_{1}\right|<\infty
$$

If $p<1$, then

$$
\sum_{j=1}^{\infty} j\left|c_{j}\right|^{p}<\infty \Rightarrow \sum_{k=0}^{\infty}\left|\tilde{c}_{k}\right|^{p}<\infty .
$$

Again, it is worth mentioning that sufficient conditions for the correctness of definition of $\tilde{\varepsilon}_{t}$ are $\sum_{k=0}^{\infty}\left|\tilde{c}_{k}\right|<\infty$ and $\mathbf{E}\left|\varepsilon_{0}\right|<\infty$. It is important that this decomposition can be applied to an arbitrary sequence $\left\{\varepsilon_{i}\right\}$ (even stationarity is not needed, the only requirement is that $X(t)$ and a new linear process $\tilde{\varepsilon}_{t}$ are correctly defined). From (2) it follows that

$$
a_{n}^{-1} \sum_{t=1}^{n} X_{t}=C_{1} a_{n}^{-1} \sum_{t=1}^{n} \varepsilon_{t}+a_{n}^{-1}\left(\tilde{\varepsilon}_{0}-\tilde{\varepsilon}_{n}\right),
$$

where $a_{n}, n \geqslant 1$, is some sequence of normalizing constants. Having (3), the next step is to prove, under appropriate moment conditions on $\left\{\varepsilon_{i}\right\}$ (which usually are assumed to be i.i.d. or martingale differences) and some conditions on coefficients $c_{i}$, that $a_{n}^{-1}\left(\tilde{\varepsilon}_{0}-\tilde{\varepsilon}_{n}\right) \rightarrow 0$ in probability or a.s. Thus limit theorems for $\sum_{t=1}^{n} X_{t}$ are reduced to corresponding limit theorems for $\sum_{t=1}^{n} \varepsilon_{t}$.

$\mathrm{R} \mathrm{e} \mathrm{m} \mathrm{a} \mathrm{r} \mathrm{k} \mathrm{1.} \mathrm{This} \mathrm{remark} \mathrm{concerns} \mathrm{the} \mathrm{relation} \mathrm{(2)} \mathrm{and} \mathrm{the} \mathrm{term}$ BND. It is not difficult to see (but it seems that this fact was not mentioned 
in any paper dealing with BND) that the relation (2) is nothing else but the so-called Abel transformation (or summation by parts, a discrete analogue of integration by parts) which usually is stated as follows: for any numbers $a_{k}, b_{k}, k=0,1, \ldots, n$,

$$
\sum_{k=0}^{n} a_{k} b_{k}=a_{0} \sum_{k=0}^{n} b_{k}+\sum_{j=0}^{n-1}\left(a_{j+1}-a_{j}\right) \sum_{k=j+1}^{n} b_{k},
$$

or, assuming that all series, obtained by passing to limit $n \rightarrow \infty$, are convergent, we can write

$$
\sum_{k=0}^{\infty} a_{k} b_{k}=a_{0} \sum_{k=0}^{\infty} b_{k}+\sum_{j=0}^{\infty}\left(a_{j+1}-a_{j}\right) \sum_{k=j+1}^{\infty} b_{k} .
$$

Taking $b_{k}=c_{k}, a_{k}=\varepsilon_{t-k}$, from (4) we get (2). Although in [9] it is said that identity (2) in the context of time series was known and used before the paper [3], the abbreviation BN decomposition was used (maybe for the first time) in [9]. Now this term Beveridge-Nelson decomposition is so widely spread in econometric literature (Google Scholar gives more than 1400 entries connected with this term), that to return Abel's name for this simple algebraic identity would be impossible. Moreover, the term BND reflects the credit given to the authors who gave the economical interpretation for this algebraic identity as decomposition of time series into permanent and transitory components and relating them with «business cycles».

$\mathrm{R}$ e $\mathrm{m}$ a $\mathrm{r} \mathrm{k} 2$. The second remark concerns the relation between BND and Gordin approach. In the paper [9] it is mentioned that «the BND approach is related to the method pioneered by Gordin [5] for developing central limit theorems for stationary processes via corresponding results for approximating martingales». We can say more. If we take (1) with i.i.d. random variables $\varepsilon_{i}, i \in \mathbf{Z}, \mathbf{E} \varepsilon_{0}=0$, and if we suppose that the series defined by $\tilde{\varepsilon}_{t}=\sum_{k=0}^{\infty} \tilde{c}_{k} \varepsilon_{t-k}$ converges, then by BND we can write

$$
\sum_{t=1}^{n} X_{t}=C_{1} \sum_{t=1}^{n} \varepsilon_{t}+\left(\tilde{\varepsilon}_{0}-\tilde{\varepsilon}_{n}\right)
$$

The first term on the right-hand side of the equality is a martingale and the second one is bounded in probability. It means that we have exactly Gordin's decomposition due to its uniqueness. The same is also true in the case, where $\left\{\varepsilon_{i}\right\}$ is a stationary sequence of martingale differences. For linear processes with more general sequences $\left\{\varepsilon_{i}\right\}$ two representations (Gordin's and BND) can be different and each of them has its advantages. For example, BND can be applied for not necessarily stationary sequence $\varepsilon_{i}, i \in \mathbf{Z}$, and admit, as it will be showed below, an analog for continuous time processes (as far as we know, Gordin's approach till now has no analogue for stationary processes in continuous time). 
The rest of the paper has a very simple structure: in Section 2 we formulate various decompositions for linear processes and fields with continuous argument and applications of these decompositions for limit theorems, while Section 3 contain proofs.

\section{Formulation of results.}

2.1. Decomposition for linear processes and fields with continuous time. Consider a linear process

$$
X(t)=\int_{0}^{\infty} \varphi(u) \varepsilon(t-u) d u
$$

where a nonrandom function $\varphi$ is integrable and such that

$$
M_{1}=\int_{0}^{\infty} \varphi(u) d u \neq 0
$$

and $\varepsilon(t), t \in \mathbf{R}$, is a stationary random process such that $X$ is well defined (for example, if $\mathbf{E}|\varepsilon(0)|<\infty$ and $\int_{0}^{\infty}|\varphi(u)| d u<\infty$ ) and, therefore, is also a stationary process. All integrals appearing in the paper are Lebesgue integrals. Intuitively it is tempting to try to express $X(t)$ as a sum of $M_{1} \varepsilon(t)$ and remainder term, involving new linear process. But since it is clear that difference operation (see the term $\tilde{\varepsilon}_{t-1}-\tilde{\varepsilon}_{t}$ in (2)) in discrete time, which can be applied to arbitrary random variables, will become differentiation operation which, contrarily, is rather restrictive, so a better idea is to start with an integral analogue of (3). We set

$$
\varphi^{*}(t)=\int_{t}^{\infty} \varphi(u) d u .
$$

Let us introduce the following condition:

$$
\mathscr{L}_{q, p}: \quad \int_{0}^{\infty} u^{q}|\varphi(u)|^{p} d u<\infty, \quad q \geqslant 0, \quad p>0 .
$$

Theorem 1. Supposing that all quantities below are correctly defined, we have that for any $T>0$ the following identity holds:

$$
\int_{0}^{T} X(t) d t=M_{1} \int_{0}^{T} \varepsilon(t) d t-(\tilde{\varepsilon}(T)-\tilde{\varepsilon}(0))
$$

where $\tilde{\varepsilon}(t)=\int_{0}^{\infty} \varphi^{*}(u) \varepsilon(t-u) d u$. The relation

$$
\int_{0}^{\infty}\left|\varphi(u)^{*}\right|^{p} d u<\infty
$$

holds if condition $\mathscr{L}_{p, p}$ in case $1 \leqslant p<\infty$ and condition $\mathscr{L}_{1, p}$ in case $p<1$ is satisfied. 
Here is one difference comparing with the decomposition (2) in discrete time. If in the case of discrete time conditions $\mathscr{L}_{p, p}$ or $\mathscr{L}_{1, p}$ guarantee the finiteness of $C_{1}$ (see Proposition 1), now these conditions guarantee only finiteness of integral $\int_{1}^{\infty}|\varphi(u)| d u$, therefore, assuming one of these conditions, we still must assume finiteness of $M_{1}$, or, more precisely, finiteness of $\int_{0}^{1}|\varphi(u)| d u$.

Having relation (6) we can get the decomposition for $X(t)$ itself, as it was mentioned above. Namely, taking $t$ instead of $T$ in (6), differentiating both sides with respect to $t$, and supposing that the obtained integrals are correctly defined (for this it is sufficient to suppose that $\int_{0}^{\infty}\left|\varphi^{*}(u)\right| d u<$ $\infty$ and $\mathbf{E}\left|\varepsilon^{\prime}(0)\right|<\infty$; these conditions justify the change of the order of differentiation and integration) we get the following decomposition:

$$
X(t)=M_{1} \varepsilon(t)-\int_{0}^{\infty} \varphi^{*}(u) \frac{d}{d t} \varepsilon(t-u) d u,
$$

but at present we cannot see how it can be used.

Relation (6) (which could be called integral BND in continuous time) shows that investigation of asymptotic properties of integral $\int_{0}^{T} X(t) d t$ can be reduced to the studies of these properties for $\int_{0}^{T} \varepsilon(t) d t$, if we can show that remainder term after normalization tends to zero in appropriate sense. We will demonstrate such possibility in the next subsection.

In (5) linear process is defined as a linear combination of innovation process $\varepsilon$ over the values in the «past», but it is possible to consider linear combination over all values. Namely, let

$$
X(t)=\int_{-\infty}^{\infty} \varphi(u) \varepsilon(t-u) d u,
$$

where function $\varphi$ is integrable and such that

$$
\widetilde{M}_{1}=\int_{-\infty}^{\infty} \varphi(u) d u \neq 0 .
$$

Then we have the following analogue of Theorem 1.

Proposition 2. For any $T>0$ the following identity holds:

$$
\int_{0}^{T} X(t) d t=\widetilde{M}_{1} \int_{0}^{T} \varepsilon(t) d t-\int_{-\infty}^{\infty} \tilde{\varphi}^{*}(u)(\varepsilon(T-u)-\varepsilon(-u)) d u,
$$

where

$$
\tilde{\varphi}^{*}(u)=\int_{u}^{\infty} \varphi(t) d t, \quad \text { for } u \geqslant 0, \quad \tilde{\varphi}^{*}(u)=\int_{-\infty}^{u} \varphi(t) d t, \quad \text { for } u<0 .
$$

With obvious changes the same relations between $\tilde{\varphi}^{*}(u)$ and $\varphi(u)$ as in Theorem 1 hold. 
It is interesting to note, that neither in [9] nor in [8] an analogue of (9) for discrete time series was not formulated, maybe for the reason that in time series one usually considers the so-called casual processes. For a linear process

$$
Y_{t}=\sum_{k=-\infty}^{\infty} c_{k} \varepsilon_{t-k}, \quad t \in \mathbf{Z}
$$

denoting $\mu_{1}:=\sum_{k=-\infty}^{\infty} c_{k} \neq 0$ we can write the following decomposition:

$$
Y_{t}=\mu_{1} \varepsilon_{t}-\left(\hat{\varepsilon}_{t}-\hat{\varepsilon}_{t-1}\right),
$$

where $\hat{\varepsilon}_{t}=\sum_{k=-\infty}^{\infty} c_{k}^{*} \varepsilon_{t-k}$ and

$$
c_{k}^{*}=\sum_{j=k+1}^{\infty} c_{j}, \quad \text { for } k \geqslant 0, \quad c_{k}^{*}=-\sum_{j=-\infty}^{k} c_{j}, \quad \text { for } k<0 .
$$

Now we consider linear random fields. In [8] (see also [7]) BND for discrete random linear fields was stated and some applications of this decomposition for limit theorems was demonstrated. Having decomposition (6) for linear processes with continuous time, it became clear that the generalization to linear random fields is available. Since the complexity of such decomposition grows rather rapidly with dimension of argument (see [8]), we restrict ourselves with the two-dimensional case. Now bold letters stand for vectors: $\mathbf{T}=\left(T_{1}, T_{2}\right), \mathbf{t}=\left(t_{1}, t_{2}\right), d \mathbf{t}=d t_{1} d t_{2}, \mathbf{1}_{A}$ denotes an indicator function of a set $A,|\mathbf{T}|=T_{1} T_{2}$ and $\mathbf{T} \rightarrow \infty$ means that $\min \left(T_{1}, T_{2}\right) \rightarrow \infty$. The product of two vectors is meant coordinate-wise, $\mathbf{t T}=\left(t_{1} T_{1}, t_{2} T_{2}\right)$. We denote $[\mathbf{0}, \mathbf{T}]:=\left[0, T_{1}\right] \times\left[0, T_{2}\right]$ and we always assume that $T_{i}>0, i=1,2$, since integrals of stationary processes over more general rectangles can be expressed as a sum of integrals over rectangles with $T_{i}>0, i=1,2$. Also $[\mathbf{0}, \bar{\infty})$ will stand for $[0, \infty) \times[0, \infty)$ and, similarly, $(-\bar{\infty}, \bar{\infty})$ will stand for $(-\infty, \infty) \times(-\infty, \infty)$.

Let us consider a linear random field

$$
X(\mathbf{t})=\int_{[\mathbf{0}, \bar{\infty})} \varphi(\mathbf{u}) \varepsilon(\mathbf{t}-\mathbf{u}) d \mathbf{u} .
$$

Here a function $\varphi$ is integrable and such that

$$
M_{11}=\int_{[\mathbf{0}, \bar{\infty})} \varphi(\mathbf{u}) d \mathbf{u} \neq 0,
$$

$\varepsilon(\mathbf{t}), \mathbf{t} \in \mathbf{R}^{2}$, is a mean zero stationary random field such that $X$ is well defined and, therefore, is a stationary field. Let us denote (assuming, of course, that all integrals in the formulae below are well defined)

$$
V(\mathbf{t})=\int_{[\mathbf{0}, \bar{\infty})} \varphi^{*}(\mathbf{u}) \varepsilon(\mathbf{t}-\mathbf{u}) d \mathbf{u}, \quad \varphi^{*}(\mathbf{s})=\int_{[\mathbf{s}, \bar{\infty})} \varphi(\mathbf{u}) d \mathbf{u},
$$




$$
\begin{array}{ll}
U_{1}(\mathbf{t})=\int_{0}^{t_{2}} \int_{0}^{\infty} g\left(u_{1}\right) \varepsilon\left(t_{1}-u_{1}, u_{2}\right) d \mathbf{u}, & g(u)=\varphi^{*}(u, 0), \\
U_{2}(\mathbf{t})=\int_{0}^{t_{1}} \int_{0}^{\infty} h\left(u_{2}\right) \varepsilon\left(u_{1}, t_{2}-u_{2}\right) d \mathbf{u}, & h(u)=\varphi^{*}(0, u) .
\end{array}
$$

Theorem 2. We suppose that all quantities defined above are correctly defined, then for any $\mathbf{T}>0$ the following identity holds:

$$
\int_{[\mathbf{0}, \mathbf{T}]} X(\mathbf{t}) d \mathbf{t}=M_{11} \int_{[\mathbf{0}, \mathbf{T}]} \varepsilon(\mathbf{t}) d \mathbf{t}+R(\mathbf{T})
$$

where

$$
\begin{aligned}
R(\mathbf{T}) & =R_{1}(\mathbf{T})-R_{2}(\mathbf{T}), \\
R_{1}(\mathbf{T}) & =V(\mathbf{T})-V\left(0, T_{2}\right)-V\left(T_{1}, 0\right)+V(0,0), \\
R_{2}(\mathbf{T}) & =U_{1}(\mathbf{T})-U_{1}\left(0, T_{2}\right)+U_{2}(\mathbf{T})-U_{2}\left(T_{1}, 0\right) .
\end{aligned}
$$

Relations between integrability of $\varphi^{*}(\mathbf{u})$ and $\varphi(\mathbf{u})$, analogous to relations in discrete case, can easily be stated. Having relation (11) one can use it to prove limit theorems for integrals of a linear random field, as it was done in discrete case in [8]. One such application will be demonstrated in Theorem 5 .

As in the case of linear processes (see (8) and Proposition 2) it is possible to consider linear random fields of the form

$$
X(\mathbf{t})=\int_{(-\bar{\infty}, \bar{\infty})} \varphi(\mathbf{u}) \varepsilon(\mathbf{t}-\mathbf{u}) d \mathbf{u},
$$

and, assuming that

$$
\widetilde{M}_{11}=\int_{(-\bar{\infty}, \bar{\infty})} \varphi(\mathbf{u}) d \mathbf{u} \neq 0
$$

to formulate an analogue of BND for linear field (12):

$$
\int_{[\mathbf{0}, \mathbf{T}]} X(\mathbf{t}) d \mathbf{t}=\widetilde{M}_{11} \int_{[\mathbf{0}, \mathbf{T}]} \varepsilon(\mathbf{t}) d \mathbf{t}+\widetilde{R}(\mathbf{T}) .
$$

Since we do not intend to use explicit form of the remainder $\tilde{R}(\mathbf{T})$, we do not provide what changes comparing with expression in (11) one must make (main change is in definition of the function $\varphi^{*}(\mathbf{u})$ ).

2.2. Limit theorems. Let $\{Y(t), t \in \mathbf{R}\}$, be a mean zero stationary process. We say that the Strong Law of Large Numbers (SLLN) holds for $Y$, if

$$
T^{-1} \int_{0}^{T} Y(t) d t \stackrel{\text { a.s. }}{\longrightarrow} 0 \text {. }
$$


Assuming additionally that $\sigma^{2}=\mathbf{E} Y^{2}(t)<\infty$, we say that the Central Limit Theorem (CLT) holds for $Y$ with normalizing function $a(T) \rightarrow \infty$ as $T \rightarrow \infty$, if

$$
a(T)^{-1} \int_{0}^{T} Y(t) d t \stackrel{d}{\longrightarrow} N(0,1)
$$

where $N(0,1)$ stands for the standard normal law. We say that the Invariance Principle (IP) for $Y$ holds with normalizing function $a(T)$, if the process

$$
Y_{T}(t):=a(T)^{-1} \int_{0}^{t T} Y(u) d u, \quad t \in[0,1],
$$

converges weakly in the Banach space $C[0,1]$ to some Gaussian process.

General scheme of application of decomposition (6) in continuous time is the following: assuming that one of the laws formulated above holds for the process $\varepsilon(t)$, one must show that the remainder term tends to zero in probability (for CLT and IP) or a.s. (for SLLN), thus getting the corresponding statement for the process $X$. Concerning SLLN one can note that application of (6) is almost useless, since usually to get SLLN for the process $\varepsilon(t)$, we assume that it is mean zero ergodic and stationary, but then the same properties has the process $X(t)$ and SLLN for it holds (see [2], where SLLN is considered for discrete random fields). We provide only three examples of application of decompositions formulated in the previous subsection. In Theorem 3 we prove the IP, in Theorem 4 we consider processes with infinite variance, and in Theorem 5 we prove the CLT for fields without using the explicit form of the remainder term in (11).

Theorem 3. Suppose that $X(t)$ is defined in (5). Let IP hold for $\varepsilon(t)$ with $a(T)=C T^{-1 / 2}$, where $C$ is a positive constant, and let $\varphi$ satisfy the condition $\mathscr{L}_{1,1}$. Then IP holds for $X(t)$ with the same normalizing function.

$\mathrm{R}$ e $\mathrm{m}$ a r k 3 . Since the CLT follows from IP (but, generally, not converse), from this result we get the CLT for linear random processes. In the proof of Theorem 3, explicit form of the remainder term in (6) is used. From discrete case we know (see [9]) that for CLT proved by the so-called indirect application of BND the condition $\mathscr{L}_{1,1}$ is superfluous. The same situation is in the case of continuous time processes, and the CLT for linear random processes holds under weaker condition on a function $\varphi$, see Remark 5 .

$\mathrm{R} \mathrm{e} \mathrm{m}$ a r k 4 . In the monograph [6], the whole Chapter XVIII is devoted for the CLT for sums or integrals of a stationary random processes

$$
B(T)^{-1} \sum_{t=1}^{T} \varepsilon_{t}-A(T), \quad B(T)^{-1} \int_{0}^{T} \varepsilon(t) d t-A(T),
$$

where $B(T)$ and $A(T)$ are normalizing and centering constants and $\left(\varepsilon_{k}, k \in\right.$ $\mathbf{Z})$ and $(\varepsilon(t), t \in \mathbf{R})$ are stationary processes satisfying some mixing type 
conditions. Verification of mixing type conditions for linear processes and, especially, for fields is not an easy task, therefore our results obtained by using representation (6) or (11) can be considered as complementary to the results from [6]: if we are able to verify that the innovations process $\varepsilon(t)$, $t \in \mathbf{Z}$, satisfies conditions formulated in [6], then applying theorems from our paper we get the CLT for linear processes or fields.

Theorem 4. Suppose we have linear process (5) and $\varepsilon(t), t \in \mathbf{R}$, is a stationary random process with $\mathbf{E} \varepsilon(0)=0$, if it exists, and such that the random process

$$
\tilde{\varepsilon}(t)=\int_{0}^{\infty} \varphi^{*}(u) \varepsilon(t-u) d u
$$

is well defined and is stationary. Assume that as $T \rightarrow \infty$

$$
T^{-1 / \alpha} \int_{0}^{T} \varepsilon(u) d u \stackrel{d}{\longrightarrow} \eta,
$$

where $\eta$ is some stable random variable with the exponent $0<\alpha<2$. If $M_{1} \neq 0$, then

$$
T^{-1 / \alpha} \int_{0}^{T} X(u) d u \stackrel{d}{\longrightarrow} M_{1} \eta
$$

as $T \rightarrow \infty$.

For simplicity of formulation the normalization by $T^{-1 / \alpha}$ is used in (15) and (16), but, clearly, one can formulate the result with a more general normalization involving slowly varying functions. Essentially condition (14) ensures that the remainder term in BND after appropriate normalization tends to zero in probability. In the case, where $\mathbf{E}|\varepsilon(0)|$ is finite, the sufficient condition for $(14)$ is $\mathscr{L}_{1,1}$, since we can estimate

$$
\mathbf{E}|\tilde{\varepsilon}(t)| \leqslant \mathbf{E}|\varepsilon(0)| \int_{0}^{\infty}\left|\varphi^{*}(u)\right| d u
$$

and apply (7) with $p=1$. We conjecture that (15) and some condition on the function $\varphi$ imply (14), but to prove this we need to know what properties of a stationary process $\varepsilon(t), t \in \mathbf{R}$, ensures (15). In the case of infinite mean of $\varepsilon(0)$ we can propose the following sufficient condition for (14).

Proposition 3. Let $0<\alpha \leqslant 1$. Assume that $\varphi$ is integrable, $\mathscr{L}_{1, p}$ with $p=\alpha /(1+\alpha)$ holds, and the following condition take place:

$$
\mathbf{E} \xi_{1}^{\alpha}<\infty
$$

where $\xi_{1}=\sup _{t \in[0,1]}|\varepsilon(t)|$. Then for all the integral $\int_{0}^{\infty} \varphi^{\star}(u) \varepsilon(t-u) d u$ is defined a.s. 
As the third example of an application of the decomposition we formulate the CLT for linear random fields. Suppose that $\varepsilon(\mathbf{t}), \mathbf{t} \in \mathbf{R}^{2}$, is a mean zero stationary random field with finite second moment $\sigma_{0}^{2}=\mathbf{E} \varepsilon^{2}(\mathbf{0})$ and covariance function $\rho(\mathbf{t})=\mathbf{E} \varepsilon(\mathbf{0}) \varepsilon(\mathbf{t})$. We assume that the following condition is satisfied:

$$
\int_{(-\bar{\infty}, \bar{\infty})}|\rho(\mathbf{t})| d \mathbf{t}<\infty
$$

therefore the following limit exists:

$$
\sigma_{1}^{2}=\lim _{\mathbf{T} \rightarrow \infty} \int_{[-\mathbf{T}, \mathbf{T}]} \rho(\mathbf{t})\left(1-\frac{\left|t_{1}\right|}{T_{1}}\right)\left(1-\frac{\left|t_{2}\right|}{T_{2}}\right) d \mathbf{t}=\int_{(-\bar{\infty}, \bar{\infty})} \rho(\mathbf{t}) d \mathbf{t} .
$$

We assume that for random field $\varepsilon(\mathbf{t}), \mathbf{t} \in \mathbf{R}^{2}$, the CLT holds, that is, if $\mathbf{T} \rightarrow \infty$, then

$$
(2 \sqrt{|\mathbf{T}|})^{-1} \int_{[-\mathbf{T}, \mathbf{T}]} \varepsilon(\mathbf{t}) d \mathbf{t} \stackrel{d}{\longrightarrow} N\left(0, \sigma_{1}^{2}\right) .
$$

It is possible to consider more general rectangles $\left[-\mathbf{T}_{1}, \mathbf{T}_{2}\right]$ with $\mathbf{T}_{i} \geqslant \mathbf{0}$ (in particular, to take $\mathbf{T}_{1}=\mathbf{0}$ ), but in the case of symmetric set $[-\mathbf{T}, \mathbf{T}]$ some expressions in the proof are simpler.

Theorem 5. Suppose that (18) holds and $\widetilde{M}_{11} \neq 0$. Then the CLT for the linear field defined in (12) holds:

$$
(2 \sqrt{|\mathbf{T}|})^{-1} \int_{[-\mathbf{T}, \mathbf{T}]} X(\mathbf{t}) d \mathbf{t} \stackrel{d}{\longrightarrow} N\left(0, \sigma_{2}^{2}\right),
$$

where $\sigma_{2}^{2}=\sigma_{1}^{2} \widetilde{M}_{11}^{2}$.

$\mathrm{R} \mathrm{e} \mathrm{ma} \mathrm{r} \mathrm{k} \mathrm{5.} \mathrm{From} \mathrm{the} \mathrm{proof} \mathrm{of} \mathrm{Theorem} 5$ and Lemma 2 one can see that with obvious changes the same result holds for linear processes, too. We note, that CLT for linear processes, which follows from IP formulated in Theorem 3, requires stronger condition on the function $\varphi$, since in the proof a direct application of decomposition is used, while the above formulated theorem is proved using indirect application of this decomposition.

Ending this section we would like to note that we had considered continuous linear processes given by the simplest form (5). The next step will be to consider linear processes of the form

$$
X(t)=\int_{-\infty}^{t} \varphi(t-u) d \varepsilon(u)
$$

with appropriately defined stochastic integral with respect to the process $\varepsilon$. For example, taking a Lévy process as $\varepsilon$ we shall arrive at the so-called CARMA (continuous autoregressive moving average) processes, which are important in financial time series theory, see, for example, [4] or [1], where 
one can find many survey papers devoted to CARMA processes. Finally, even for processes (5) we leave some questions open, for example, we do not know if it is possible to prove limit theorems for integrals

$$
\int_{0}^{T}|X(t)|^{2} d t
$$

using decomposition (6), like it was done in [9] for discrete time series.

\section{Proofs.}

P r o of of $\mathrm{Th}$ e or e $\mathrm{m} 1$. The proof of (6) is very simple, namely, using the integration by parts (this is justified by the fact that both functions $\varphi^{*}(u)$ and $\int_{-u}^{T-u} \varepsilon(s) d s$ are differentiable with respect to $u$ ), we can write

$$
\begin{aligned}
\int_{0}^{\infty} & \varphi^{*}(u)(\varepsilon(T-u)-\varepsilon(-u)) d u=\int_{0}^{\infty} \varphi^{*}(u) d\left(\int_{-u}^{T-u} \varepsilon(s) d s\right) \\
& =\left.\varphi^{*}(u) \int_{-u}^{T-u} \varepsilon(s) d s\right|_{0} ^{\infty}+\int_{0}^{\infty} \varphi(u) \int_{-u}^{T-u} \varepsilon(s) d s d u \\
& =-\int_{0}^{\infty} \varphi(u) d u \int_{0}^{T} \varepsilon(s) d s+\int_{0}^{T}\left(\int_{0}^{\infty} \varphi(u) \varepsilon(s-u) d u\right) d s \\
& =-M_{1} \int_{0}^{T} \varepsilon(t) d t+\int_{0}^{T} X(t) d t
\end{aligned}
$$

and this equality coincides with (6).

Let us prove (7). If $0<p \leqslant 1$, then

$$
\int_{0}^{\infty}\left|\varphi(u)^{*}\right|^{p} d u \leqslant \int_{0}^{\infty} \int_{u}^{\infty}|\varphi(s)|^{p} d s d u=\int_{0}^{\infty} s|\varphi(s)|^{p} d s .
$$

If $p>1$, then standard application of the Hölder inequality with some $\alpha$, satisfying $1 / q<\alpha<1 / q+1 / p=1$, yields

$$
\begin{aligned}
\int_{0}^{\infty}\left|\varphi(u)^{*}\right|^{p} d u & =\int_{0}^{\infty}\left|\int_{u}^{\infty} s^{\alpha} \varphi(s) s^{-\alpha} d s\right|^{p} d u \\
& \leqslant \int_{0}^{\infty}\left(\int_{u}^{\infty} s^{\alpha p}|\varphi(s)|^{p} d s\right)\left(\int_{u}^{\infty} s^{-\alpha q} d s\right)^{p / q} d u \\
& =\frac{1}{q \alpha-1} \int_{0}^{\infty} u^{(-q \alpha+1) p / q} \int_{u}^{\infty} s^{\alpha p}|\varphi(s)|^{p} d s d u \\
& =\frac{1}{q \alpha-1} \int_{0}^{\infty} s^{\alpha p}|\varphi(s)|^{p} \int_{0}^{s} u^{(-q \alpha+1) p / q} d u d s \\
& =\frac{1}{(q \alpha-1) p(1-\alpha)} \int_{0}^{\infty} s^{p}|\varphi(s)|^{p} d s
\end{aligned}
$$

The theorem is proved. 
Proof of Proposition 2. To prove (9), we write $X(t)=$ $X_{1}(t)+X_{2}(t)$, where

$$
X_{1}(t)=\int_{0}^{\infty} \varphi(-u) \varepsilon(t+u) d u, \quad X_{2}(t)=\int_{0}^{\infty} \varphi(u) \varepsilon(t-u) d u .
$$

For $X_{2}(t)$ we apply (6) and get

$$
\int_{0}^{T} X_{2}(t) d t=M_{12} \int_{0}^{T} \varepsilon(t) d t-\int_{0}^{\infty} \varphi_{2}^{*}(u)(\varepsilon(T-u)-\varepsilon(-u)) d u
$$

with

$$
M_{12}=\int_{0}^{\infty} \varphi(u) d u, \quad \varphi_{2}^{*}(u)=\int_{u}^{\infty} \varphi(t) d t .
$$

For $X_{1}(t)$, repeating the proof of (6), one can verify that the following identity holds:

$$
\int_{0}^{T} X_{1}(t) d t=M_{11} \int_{0}^{T} \varepsilon(t) d t+\int_{0}^{\infty} \varphi_{1}^{*}(u)(\varepsilon(T+u)-\varepsilon(u)) d u
$$

with

$$
M_{11}=\int_{0}^{\infty} \varphi(-u) d u, \quad \varphi_{1}^{*}(u)=\int_{u}^{\infty} \varphi(-t) d t=\int_{-\infty}^{-u} \varphi(t) d t, \quad u>0 .
$$

Changing the variables $u=-v$ in the last integral in (20) and noting that $\widetilde{M}_{1}=M_{11}+M_{12}$, we see that (19) and (20) prove (9). The proposition is proved.

P r o o f of (10). The proof is similar to the proof of Proposition 2: we write $Y_{t}=Y_{t}^{(1)}+Y_{t}^{(2)}$, where

$$
Y_{t}^{(1)}=\sum_{k=-\infty}^{0} \tilde{c}_{k} \varepsilon_{t-k}, \quad Y_{t}^{(2)}=\sum_{k=0}^{\infty} \tilde{c}_{k} \varepsilon_{t-k}
$$

and $\tilde{c}_{0}=c_{0} / 2, \tilde{c}_{k}=c_{k}$ for $k \neq 0$. For $Y_{t}^{(2)}$, applying Proposition 1, we have

$$
Y_{t}^{(2)}=\mu_{1}^{(2)} \varepsilon_{t}-(1-L) \sum_{k=0}^{\infty} \tilde{c}_{k}^{*} \varepsilon_{t-k}
$$

where $\mu_{1}^{(2)}=\sum_{k=0}^{\infty} \tilde{c}_{k}$ and $\tilde{c}_{k}^{*}=\sum_{j=k+1}^{\infty} \tilde{c}_{j}, k \geqslant 0$. Rewriting $Y_{t}^{(1)}=$ $\sum_{k=0}^{\infty} \hat{c}_{k} \varepsilon_{t+k}$ with $\hat{c}_{0}=c_{0} / 2, \hat{c}_{k}=c_{-k}$ for $k>0$, and repeating the proof of Proposition 1, one can check that

$$
Y_{t}^{(1)}=\mu_{1}^{(1)} \varepsilon_{t}-\left(1-L^{-1}\right) \sum_{k=0}^{\infty} \hat{c}_{k}^{*} \varepsilon_{t+k}
$$

where $\mu_{1}^{(1)}=\sum_{k=0}^{\infty} \hat{c}_{k}, \hat{c}_{k}^{*}=\sum_{j=k+1}^{\infty} \hat{c}_{j}, k \geqslant 0$, and $L^{-1} \varepsilon_{t}=\varepsilon_{t+1}$. Now it is not difficult to verify that (21) and (22) gives us (10). 
Proof of $\mathrm{Th}$ e or e $\mathrm{m}$ 2. For any $\mathbf{x} \in \mathbf{R}^{2}$ introduce a function

$$
A(\mathbf{x})=\int_{[\mathbf{0}, \mathbf{x}]} \varepsilon(\mathbf{u}) d \mathbf{u} .
$$

Changing the order of integration and using the introduced function $A$ and change of variables, we can rewrite the left-hand side of (11) as

$$
\begin{aligned}
\int_{[\mathbf{0}, \mathbf{T}]} X(\mathbf{t}) d \mathbf{t} & =\int_{[\mathbf{0}, \bar{\infty}]} \varphi(\mathbf{u}) \int_{[\mathbf{0}, \mathbf{T}]} \varepsilon(\mathbf{t}-\mathbf{u}) d \mathbf{t} d \mathbf{u} \\
& =\int_{[\mathbf{0}, \bar{\infty}]} \varphi(\mathbf{u}) B(\mathbf{T}, \mathbf{u}) d \mathbf{u},
\end{aligned}
$$

where

$$
B(\mathbf{T}, \mathbf{u})=A(\mathbf{T}-\mathbf{u})-A\left(T_{1}-u_{1},-u_{2}\right)-A\left(-u_{1}, T_{2}-u_{2}\right)+A\left(-u_{1},-u_{2}\right) .
$$

Now one must show that the right-hand side of (11), which is

$$
M_{11} \int_{[\mathbf{0}, \mathbf{T}]} \varepsilon(\mathbf{t}) d \mathbf{t}+R_{1}(\mathbf{T})-R_{2}(\mathbf{T}),
$$

can be transformed into the same expression, written above. The first term is a simple one:

$$
M_{11} \int_{[\mathbf{0}, \mathbf{T}]} \varepsilon(\mathbf{t}) d \mathbf{t}=\int_{[\mathbf{0}, \bar{\infty}]} \varphi(\mathbf{u}) A(\mathbf{T}) d \mathbf{u} .
$$

All terms in remainder $R_{1}(T, S)-R_{2}(T, S)$ can be treated similarly — it is sufficient to use change of the order of integration and change of variables, for example,

$$
\begin{aligned}
U_{1}(\mathbf{T}) & =\int_{0}^{T_{2}} \int_{0}^{\infty} g\left(u_{1}\right) \varepsilon\left(T_{1}-u_{1}, u_{2}\right) d \mathbf{u} \\
& =\int_{0}^{T_{2}} \int_{0}^{\infty} \int_{u_{1}}^{\infty} \int_{0}^{\infty} \varphi(\mathbf{s}) d \mathbf{s} \varepsilon\left(T_{1}-u_{1}, u_{2}\right) d \mathbf{u} \\
& =\int_{\mathbf{0}}^{\infty} \varphi(\mathbf{s}) \int_{0}^{s_{1}} \int_{0}^{T_{2}} \varepsilon\left(T_{1}-u_{1}, u_{2}\right) d \mathbf{u} d \mathbf{s} \\
& =\int_{\mathbf{0}}^{\infty} \varphi(\mathbf{s})\left\{A(\mathbf{T})-A\left(T_{1}-s_{1}, T_{2}\right)\right\} d \mathbf{s} \\
& =\int_{0}^{\infty} \varphi(\mathbf{u})\left\{A(\mathbf{T})-A\left(T_{1}-u_{1}, T_{2}\right)\right\} d \mathbf{u} .
\end{aligned}
$$

(Here we recall that $A(\mathbf{x})=0$ if at least one coordinate of the argument is 0.) Although we need to consider each of integrals on the right-hand side of (11), there is a lot of cancelations and finally we see that we get the same expression which was given in (23). The theorem is proved. 
$\mathrm{Pr}$ o of of $\mathrm{Th}$ e or e m 3. Without loss of generality we may assume that $C=1$ in the normalizing constant $a(T)$. Let us denote

$$
X_{T}(t):=T^{-1 / 2} \int_{0}^{t T} X(u) d u, \quad t \in[0,1] .
$$

Using (6), we can write

$$
X_{T}(t)=T^{-1 / 2}\left(M_{1} \int_{0}^{t T} \varepsilon(u) d u-\int_{0}^{\infty} \varphi^{*}(u)(\varepsilon(t T-u)-\varepsilon(-u)) d u\right) .
$$

Since according to the assumption of the proposition the IP for $\varepsilon(t)$ holds, to prove the proposition we need to show that as $T \rightarrow \infty$

$$
T^{-1 / 2} \sup _{0 \leqslant t \leqslant 1}\left|\int_{0}^{\infty} \varphi^{*}(u)(\varepsilon(t T-u)-\varepsilon(-u)) d u\right| \stackrel{\mathbf{P}}{\longrightarrow} 0 .
$$

It is clear that we can consider only integer values of $T$, that is, we need to show that

$$
n^{-1 / 2} \sup _{0 \leqslant t \leqslant n}\left|\int_{0}^{\infty} \varphi^{*}(u)(\varepsilon(t-u)-\varepsilon(-u)) d u\right| \stackrel{\mathbf{P}}{\longrightarrow} 0 .
$$

Due to the stationarity of the process $\varepsilon$ and the condition $\mathscr{L}_{1}$

$$
\mathbf{E}\left|\int_{0}^{\infty} \varphi^{*}(u) \varepsilon(-u) d u\right|<\infty,
$$

therefore

$$
n^{-1 / 2}\left|\int_{0}^{\infty} \varphi^{*}(u) \varepsilon(-u) d u\right| \stackrel{\mathbf{P}}{\longrightarrow} 0,
$$

and it remains to prove

$$
n^{-1 / 2} \sup _{0 \leqslant t \leqslant n}\left|\int_{0}^{\infty} \varphi^{*}(u) \varepsilon(t-u) d u\right| \stackrel{\mathbf{P}}{\longrightarrow} 0 .
$$

Let us denote

$$
x_{k}=\sup _{k-1 \leqslant t \leqslant k}|\tilde{\varepsilon}(t)|, \quad k \in \mathbf{Z} .
$$

Recalling that $\tilde{\varepsilon}(t)=\int_{0}^{\infty} \varphi^{*}(u) \varepsilon(t-u) d u$ is a stationary process, we can deduce that $\left\{x_{k}, k \in \mathbf{Z}\right\}$ is a stationary sequence and $\mathbf{E} x_{1}^{2}<\infty$. We provide the proof of this last assertion. After a change of variables we can write

$$
\tilde{\varepsilon}(t)=\int_{-\infty}^{t} \varphi^{*}(t-u) \varepsilon(u) d u
$$

hence

$$
x_{1}=\sup _{0 \leqslant t \leqslant 1}|\tilde{\varepsilon}(t)| \leqslant \sup _{0 \leqslant t \leqslant 1} \int_{R}\left|\varphi^{*}(t-u)\right| \mathbf{1}_{(-\infty, t]}(u)|\varepsilon(u)| d u .
$$


It is easy to see that, for $t \in[0,1]$,

$$
\varphi^{*}(t-u) \mid \mathbf{1}_{(-\infty, t]}(u) \leqslant \Psi_{1}(u)+\Psi_{2}(u),
$$

where

$$
\Psi_{1}(u)=\left|\varphi^{*}(t-u)\right| \mathbf{1}_{(-\infty, 1]}(u), \quad \Psi_{2}(u)=\int_{-u}^{-u+1}|\varphi(s)| d s .
$$

Here one must explain how to understand $\varphi^{*}(-u)$ for positive $u$. Since we deal with linear processes defined in (5), we may assume that $\varphi(s)=0$ for $s<$ 0 and then $\varphi^{*}(-u)=M_{1}$ for all $u>0$ (therefore we leave indicator function in expression of $\Psi_{1}(u)$; another possibility is to redefine $\varphi(s)=\varphi(-s)$ and then to define $\varphi^{*}(u)$ as in Proposition 2). Now writing $x_{1} \leqslant I_{1}+I_{2}$, where

$$
I_{i}=\int_{\mathbf{R}} \Psi_{i}(u)|\varepsilon(u)| d u, \quad i=1,2,
$$

and noting that both functions $\Psi_{i}(u), i=1,2$ are integrable, we easily get $\mathbf{E} I_{i}^{2}<\infty, i=1,2$, therefore $\mathbf{E} x_{1}^{2}<\infty$.

It is easy to see that (24) follows if we prove

$$
n^{-1} \max _{1 \leqslant k \leqslant n} x_{k}^{2} \stackrel{\mathbf{P}}{\longrightarrow} 0,
$$

and this relation is a consequence of the following simple lemma.

Lemma 1. Let $z_{k}, k \in \mathbf{Z}$, be a stationary sequence of nonnegative random variables and $\mathbf{E} z_{1}=a<\infty$. Then

$$
n^{-1} \max _{1 \leqslant k \leqslant n} z_{k} \stackrel{L_{1}}{\longrightarrow} 0 .
$$

$\mathrm{Pr}$ o of of $\mathrm{L}$ e m m a 1. Most probably this is a well-known fact, but since sometimes it is more difficult to find relevant reference than to prove the statement, we provide several lines of the proof of this simple lemma. For any $\delta>0$ the following inequality holds a.s.:

$$
K_{n}:=n^{-1} \max _{1 \leqslant k \leqslant n} z_{k} \leqslant n^{-1} \sum_{k=1}^{n} z_{k} \mathbf{1}_{\left\{z_{k}>n \delta\right\}}+\delta,
$$

hence

$$
\mathbf{E} K_{n} \leqslant \int_{n \delta}^{\infty} x F_{z_{1}}(d x)+\delta .
$$

This means that $\lim _{n} \mathbf{E} K_{n} \leqslant \delta$, and since $\delta$ can be chosen arbitrarily small, this proves the lemma.

Thus, we have proved (24), and Theorem 3 is proved.

P r o of of $\mathrm{Th}$ e o r e $\mathrm{m}$ 4. The proof is almost trivial: applying (6), we need to show that the remainder term multiplied by $T^{-1 / \alpha}$ converges 
in probability to zero. This follows from $(14)$, since $\tilde{\varepsilon}(t)$ is bounded in probability.

Pro of of Proposition 3. By stationarity of $\varepsilon(t)$ it is sufficient to study the convergence of the integral

$$
I=\int_{0}^{\infty}\left|\varphi^{\star}(u)\right||\varepsilon(u)| d u .
$$

It is clear that

$$
I \leqslant \sum_{k=0}^{\infty} a_{k} \xi_{k}
$$

where $a_{k}=\int_{k}^{k+1} \psi(u) d u, \psi(u)=\int_{u}^{\infty}|\varphi(s)| d s, \xi_{k}=\sup _{t \in[k, k+1]}|\varepsilon(t)|$.

Due to the integrability of $\varphi$, the function $\psi$ is positive, nonincreasing, and $\psi(u) \rightarrow 0$ as $u \rightarrow \infty$.

Let $\left(b_{k}\right)$ be a sequence of positive numbers. We have

$$
\mathbf{P}\left\{a_{k} \xi_{k}>b_{k}\right\} \leqslant \frac{\mathbf{E}\left|\xi_{1}\right|^{\alpha}}{b_{k}^{\alpha} a_{k}^{-\alpha}}=C_{\alpha}\left(a_{k} b_{k}^{-1}\right)^{\alpha} .
$$

It is easy to see that condition $\mathscr{L}_{1, p}$ with $p=\alpha /(1+\alpha)$ implies

$$
\int_{0}^{\infty} \psi(u)^{\alpha /(1+\alpha)} d u<\infty
$$

hence, due to the monotonicity of $\psi$, the convergence of the series $\sum_{k} a_{k}^{\alpha /(1+\alpha)}$ follows. Taking $b_{k}=a_{k}^{\alpha /(1+\alpha)}$, we get the convergence of the series $\sum_{k} b_{k}$, and from (25) it follows that

$$
\sum_{k} \mathbf{P}\left\{a_{k} \xi_{k}>b_{k}\right\}<\infty .
$$

Applying the Borel-Cantelli lemma, we get that the series $\sum_{k} a_{k} \xi_{k}$ converges a.s., and the proposition is proved.

$\mathrm{Pr}$ o of of $\mathrm{Th}$ e or e $\mathrm{m}$. As it was mentioned before the formulation of the theorem, we indirectly apply the BND. Since we have relation (18), from (13) it is clear that the theorem will be proved if we prove

$$
|\mathbf{T}|^{-1} \mathbf{E} \gamma^{2}(\mathbf{T}) \rightarrow 0
$$

as $\mathbf{T} \rightarrow \infty$, where

$$
\gamma(\mathbf{T})=\int_{[-\mathbf{T}, \mathbf{T}]} X(\mathbf{t}) d \mathbf{t}-\widetilde{M}_{11} \int_{[-\mathbf{T}, \mathbf{T}]} \varepsilon(\mathbf{t}) d \mathbf{t} .
$$

It is not difficult to get the following expression for $\gamma$ :

$$
\gamma(\mathbf{T})=\int_{\mathbf{R}^{2}} \varepsilon(\mathbf{u}) b(\mathbf{T}, \mathbf{u}) d \mathbf{u},
$$


where

$$
b(\mathbf{T}, \mathbf{u})=\int_{[-\mathbf{T}, \mathbf{T}]} \varphi(\mathbf{t}-\mathbf{u}) d \mathbf{t}-\widetilde{M}_{11} \mathbf{1}_{\{\mathbf{u} \in[-\mathbf{T}, \mathbf{T}]\}} .
$$

Having this expression, we get

$$
J:=\mathbf{E} \gamma^{2}(\mathbf{T})=\int_{\mathbf{R}^{2}} \int_{\mathbf{R}^{2}} \rho(\mathbf{t}-\mathbf{u}) b(\mathbf{T}, \mathbf{t}) b(\mathbf{T}, \mathbf{u}) d \mathbf{t} d \mathbf{u} .
$$

Applying the Hölder inequality, we have

$$
J \leqslant J_{1} J_{2}
$$

with

$$
\begin{aligned}
& J_{1}=\left(\int_{\mathbf{R}^{2}} \int_{\mathbf{R}^{2}}|\rho(\mathbf{t}-\mathbf{u})| b(\mathbf{T}, \mathbf{t})^{2} d \mathbf{t} d \mathbf{u}\right)^{1 / 2}, \\
& J_{2}=\left(\int_{\mathbf{R}^{2}} \int_{\mathbf{R}^{2}}|\rho(\mathbf{t}-\mathbf{u})| b(\mathbf{T}, \mathbf{u})^{2} d \mathbf{t} d \mathbf{u}\right)^{1 / 2} .
\end{aligned}
$$

We can write

$$
J_{1}^{2}=\int_{\mathbf{R}^{2}} g(\mathbf{t}) b(\mathbf{T}, \mathbf{t})^{2} d \mathbf{t}, \quad g(\mathbf{t})=\int_{\mathbf{R}^{2}}|\rho(\mathbf{t}-\mathbf{u})| d \mathbf{u}=\int_{\mathbf{R}^{2}}|\rho(\mathbf{u})| d \mathbf{u},
$$

and due to assumption (17) the last integral is finite. Therefore from (27)(29) we see that (26) will follow from the relation

$$
|\mathbf{T}|^{-1} \int_{\mathbf{R}^{2}} b(\mathbf{T}, \mathbf{t})^{2} d \mathbf{t} \rightarrow 0 \quad \text { as } \mathbf{T} \rightarrow \infty .
$$

We need the following analog of Lemma 13 from [8]. Let us introduce some notation. For a signed measure $\nu$ let $|\nu|$ stand for a variation of this measure and let us denote

$$
B(\mathbf{T})=[-\mathbf{T}, \mathbf{T}], \quad A(\mathbf{T})=\mathbf{R}^{2} \backslash B(\mathbf{T}), \quad a(\mathbf{T})=|\nu|(A(\mathbf{T})) .
$$

Lemma 2. Let $\nu$ be a signed measure satisfying two conditions

$$
\begin{gathered}
\int_{\mathbf{R}^{2}} \nu(d \mathbf{u})=0, \\
\int_{\mathbf{R}^{2}}|\nu|(d \mathbf{u})<\infty .
\end{gathered}
$$

Then for any $p>1$

$$
K(\mathbf{T}):=|\mathbf{T}|^{-1} \int_{\mathbf{R}^{2}}|\nu(B(\mathbf{T})-\mathbf{t})|^{p} d \mathbf{t} \rightarrow 0 \quad \text { as } \mathbf{T} \rightarrow \infty .
$$


$\mathrm{R}$ e $\mathrm{m}$ a $\mathrm{rk}$ 6. Inspecting the proof of this lemma, one can see that if in (33) the integration is not by Lebesgue measure but with respect to the counting measure $\left(\mu(\mathbf{k})=1\right.$ for all $\left.\mathbf{k} \in \mathbf{Z}^{2}\right)$ and the signed measure $\nu$ is concentrated on $\mathbf{Z}^{2}$, then we shall get Lemma 13 from [8] (it is appropriate to note that the requirement $p \leqslant 2$ is superfluous in [8]).

P r o of of $\mathrm{L} \mathrm{e} \mathrm{m} \mathrm{m} \mathrm{a} 2$. We have

$$
K(\mathbf{T})=K_{1}+K_{2},
$$

where

$$
\begin{aligned}
& K_{1}=|\mathbf{T}|^{-1} \int_{A(2 \mathbf{T})}|\nu(B(\mathbf{T})-\mathbf{t})|^{p} d \mathbf{t}, \\
& K_{2}=|\mathbf{T}|^{-1} \int_{B(2 \mathbf{T})}|\nu(B(\mathbf{T})-\mathbf{t})|^{p} d \mathbf{t} .
\end{aligned}
$$

Taking into account that $B(\mathbf{T})-\mathbf{t} \subset A(\mathbf{T})$ for $\mathbf{t} \in A(2 \mathbf{T})$, we can estimate

$$
\begin{aligned}
K_{1} & =|\mathbf{T}|^{-1} \int_{A(2 \mathbf{T})}|\nu(B(\mathbf{T})-\mathbf{t})|^{p-1}|\nu(B(\mathbf{T})-\mathbf{t})| d \mathbf{t} \\
& \leqslant a(\mathbf{T})^{p-1}|\mathbf{T}|^{-1} \int_{A(2 \mathbf{T})}|\nu(B(\mathbf{T})-\mathbf{t})| d \mathbf{t} \\
& =a(\mathbf{T})^{p-1}|\mathbf{T}|^{-1} \int_{A(2 \mathbf{T})}\left|\int_{\mathbf{R}^{2}} \mathbf{1}_{B(\mathbf{T})-\mathbf{t}}(\mathbf{u}) \nu(d \mathbf{u})\right| d \mathbf{t} \\
& \leqslant a(\mathbf{T})^{p-1}|\mathbf{T}|^{-1} \int_{\mathbf{R}^{2}} \int_{\mathbf{R}^{2}} \mathbf{1}_{B(\mathbf{T})-\mathbf{t}}(\mathbf{u})|\nu|(d \mathbf{u}) d \mathbf{t} \\
& \leqslant a(\mathbf{T})^{p-1}|\mathbf{T}|^{-1} \int_{\mathbf{R}^{2}} \int_{\mathbf{R}^{2}} \mathbf{1}_{B(\mathbf{T})-\mathbf{t}}(\mathbf{u}) d \mathbf{u}|\nu|(d \mathbf{t}) \\
& =a(\mathbf{T})^{p-1} \cdot 4|\nu|\left(\mathbf{R}^{2}\right) .
\end{aligned}
$$

From condition (32) it follows that $a(\mathbf{T}) \rightarrow 0$ as $\mathbf{T} \rightarrow \infty$, therefore

$$
K_{1} \rightarrow 0 \quad \text { as } \mathbf{T} \rightarrow \infty .
$$

Now we estimate $K_{2}$. For $\mathbf{w}=\left(w_{1}, w_{2}\right) \in B(\mathbf{2})$ let us denote

$$
\begin{gathered}
h(\mathbf{T}, \mathbf{w})=|\nu(B(\mathbf{T})-\mathbf{w} \mathbf{T})|^{p}, \\
\mathbf{d}(\mathbf{T})=(d(\mathbf{T}), d(\mathbf{T})), \quad d(\mathbf{T})=\min \left(T_{1}, T_{2}\right), \\
\delta(\mathbf{w})= \begin{cases}\left|w_{1}\right|-1, & \text { for } \mathbf{w} \in B(\mathbf{2}) \backslash B(\mathbf{1}),\left|w_{1}\right|>1,\left|w_{2}\right| \leqslant 1, \\
\left|w_{2}\right|-1, & \text { for } \mathbf{w} \in B(\mathbf{2}) \backslash B(\mathbf{1}),\left|w_{1}\right| \leqslant 1,\left|w_{2}\right|>1, \\
\min \left(\left|w_{1}\right|-1,\left|w_{2}\right|-1\right), & \text { for } \mathbf{w} \in B(\mathbf{2}) \backslash B(\mathbf{1}),\left|w_{1}\right|>1,\left|w_{2}\right|>1, \\
\min \left(1-\left|w_{1}\right|, 1-\left|w_{2}\right|\right), & \text { for } \mathbf{w} \in B(\mathbf{1})^{\circ} .\end{cases}
\end{gathered}
$$


Here $A^{\circ}$ stands for the interior points of the set $A$. Making a change of variables $\mathbf{t}=\mathbf{w} \mathbf{T}$, we can write

$$
K_{2}=\int_{B(\mathbf{2})} h(\mathbf{T}, \mathbf{w}) d \mathbf{w}
$$

It is clear that for all $\mathbf{T}$ and $\mathbf{w}$

$$
h(\mathbf{T}, \mathbf{w}) \leqslant\left(|\nu|\left(\mathbf{R}^{2}\right)\right)^{p}<\infty .
$$

If $\mathbf{w} \in B(\mathbf{2}) \backslash B(\mathbf{1})$, then $B(\mathbf{T})-\mathbf{w} \mathbf{T} \subset A(\delta(\mathbf{w}) \mathbf{d}(\mathbf{T}))$, therefore it follows that

$$
h(\mathbf{T}, \mathbf{w}) \leqslant|| \nu|(A(\delta(\mathbf{w}) \mathbf{d}(\mathbf{T})))|^{p} \rightarrow 0 \quad \text { as } \mathbf{T} \rightarrow \infty .
$$

Similarly, if $\mathbf{w} \in B(\mathbf{1})^{\circ}$, then $\mathbf{R}^{2} \backslash(B(\mathbf{T})-\mathbf{w} \mathbf{T}) \subset A(\delta(\mathbf{w}) \mathbf{d}(\mathbf{T}))$, therefore, due to $(31)$

$$
\begin{aligned}
h(\mathbf{T}, \mathbf{w}) & =\left|\nu\left(\mathbf{R}^{2} \backslash(B(\mathbf{T})-\mathbf{w} \mathbf{T})\right)\right|^{p} \leqslant|\nu(A(\delta(\mathbf{w}) \mathbf{d}(\mathbf{T})))|^{p} \\
& \leqslant(a(\delta(\mathbf{w}) \mathbf{d}(\mathbf{T})))^{p} \rightarrow 0 .
\end{aligned}
$$

Thus, applying the Lebesgue theorem on dominated convergence, we get

$$
K_{2}=\int_{B(\mathbf{2})} h(\mathbf{T}, \mathbf{w}) d \mathbf{w} \rightarrow 0 \quad \text { as } \mathbf{T} \rightarrow \infty .
$$

Relations (34)-(36) imply (33), and the lemma is proved.

Taking $\nu(d \mathbf{x})=f(\mathbf{x}) d \mathbf{x}$, we get the following statement.

Corollary 1. Let $f$ be a function of two variables satisfying two conditions

$$
\int_{\mathbf{R}^{2}} f(\mathbf{u}) d \mathbf{u}=0, \quad \int_{\mathbf{R}^{2}}|f(\mathbf{u})| d \mathbf{u}<\infty
$$

Then for any $p>1$

$$
|\mathbf{T}|^{-1} \int_{\mathbf{R}^{2}}\left|\int_{-\mathbf{T}-\mathbf{t}}^{\mathbf{T}-\mathbf{t}} f(\mathbf{u}) d \mathbf{u}\right|^{p} d \mathbf{t} \rightarrow 0 \quad \text { as } \mathbf{T} \rightarrow \infty .
$$

Unfortunately, we cannot apply this corollary for our purpose taking $f(\mathbf{x})=\varphi(\mathbf{x})$, since the first equality in (37) will fail. In order to prove (30), we use Lemma 2 with

$$
\nu(d \mathbf{x})=\varphi(\mathbf{x}) d \mathbf{x}-\widetilde{M}_{1,1} \delta_{\mathbf{0}}(d \mathbf{x}),
$$

where $\delta_{\mathbf{0}}(\mathbf{x})$ is a measure of unit mass concentrated at point $\mathbf{0}$. It is very easy to see that conditions (31) and (32) are satisfied:

$$
\begin{aligned}
& \int_{\mathbf{R}^{2}}\left(\varphi(\mathbf{x})-\widetilde{M}_{1,1} \delta_{\mathbf{0}}(\mathbf{x})\right) d \mathbf{x}=0 \\
& \int_{\mathbf{R}^{2}}\left|\varphi(\mathbf{x})-\widetilde{M}_{1,1} \delta_{\mathbf{0}}(\mathbf{x})\right| d \mathbf{x} \leqslant 2 \int_{\mathbf{R}^{2}}|\varphi(\mathbf{x})| d \mathbf{x} .
\end{aligned}
$$


Also it is easy to verify that

$$
\int_{B(\mathbf{T})-\mathbf{u}}\left(\varphi(\mathbf{x})-\widetilde{M}_{1,1} \delta_{\mathbf{0}}(\mathbf{x})\right) d \mathbf{x}=b(\mathbf{T}, \mathbf{u}),
$$

therefore, applying (33) with $p=2$, we get (30), and Theorem 5 is proved.

Acknowledgments. The authors are grateful to anonymous Referee, whose remarks and suggestions improved the presentation of the results.

\section{СПИСОК ЛИТЕРАТУРЫ}

1. Andersen T. G., Davis R. A., Kreiß J.-P., Mikosch T. (Eds.). Handbook of Financial Time Series. Berlin: Springer, 2009, 1050 p.

2. Banys P., Davydov Yu., Paulauskas V. Remarks on the SLLN for linear random fields. - Statist. Probab. Lett., 2010, v. 80, p. 489-496.

3. Beveridge S., Nelson C.R. A new approach to decomposition of economic time series into permanent and transitory components with particular attention to measurement of the «business cycle». - J. Monetary Economics, 1981, v. 7, p. 151-174.

4. Brockwell P. J. Lévy-driven CARMA processes. - Ann. Inst. Statist. Math., 2001, v. 53, p. $113-124$.

5. Гордин М. И. О центральной предельной теореме для стационарных процессов. Докл. АН СССР, 1969, т. 188, № 4, с. 739-741.

6. Ибрагимов И.А., Линник Ю.В. Независимые и стационарно связанные величины. М.: Наука, 1965, 524 с.

7. Marinucci D., Poghosyan S. Asymptotics for linear random fields. - Statist. Probab. Lett., 2001, v. 51, p. 131-141.

8. Paulauskas $V$. On Beveridge-Nelson decomposition and limit theorems for linear random fields. - J. Multivariate Anal., 2010, v. 101, p. 621-639.

9. Phillips P. C. B., Solo V. Asymptotics for linear processes. - Ann. Statist., 1992, v. 20 , p. $971-1001$. 